\title{
Treatment Outcome and Prognostic Factors for Malignant Skin Melanoma Treated with Radical Surgery
}

\author{
Wojciech Majewski ${ }^{*}$, Karolina Stanienda ${ }^{2}$, Katarzyna Wicherska ${ }^{2}$, Rafal \\ Ulczok $^{3}$, Jerzy Wydmanski ${ }^{1}$
}

\begin{abstract}
Aim: To assess the treatment outcome in patients with malignant skin melanoma and prognostic factors for distant metastases (DM), disease-free survival (DFS) and overall survival (OS). Materials and Methods: A retrospective analysis was conducted on 113 patients with malignant skin melanoma (60 females, 53 males, average age-55 years) who were treated surgically. Primary treatment consisted of local excision. In 12 cases, it was accompanied by lymph node excision. In $93(82 \%)$ cases, radicalization was necessary, which was either local only (19 cases) or accompanied by lymph node surgery/biopsy (74 cases). Possible prognostic factors such as Clark's stage and Breslow's depth of invasion, ulceration, average tumor dimensions, lymph nodes metastases $(\mathrm{pN}+)$, gender, tumor location and primary excision margins were considered. Results: In 51 (45\%) cases, treatment failure occurred. The 5-year DM rate was 47\%, the 5-year DFS was 38\%, and the 5-year OS was $56 \%$. In the univariate analysis, the important factors with respect to at least one endpoint included Clark's stage, Breslow's depth of invasion, ulceration, average tumor dimensions, lymph nodes metastases, gender and primary tumor localization. The presence of metastasic nodes was the most important prognostic factor, with a 5-year DM rates of $30 \%$ for $\mathrm{pN}(-)$ and $76 \%$ for $\mathrm{pN}(+)$ and a 5 -year DFS and OS of $56 \%$ and $76 \%$ for $\mathrm{pN}(-)$ and $13 \%$ and $24 \%$ for $\mathrm{pN}(+)$, respectively. The average tumor dimension was independently significant for DFS and OS, with 5-year rates of $69 \%$ and $80 \%$ for $\leq 1 \mathrm{~cm}, 28 \%$ and $53 \%$ for $1-2 \mathrm{~cm}$, and $18 \%$ and $30 \%$ for $>2 \mathrm{~cm}$, respectively. Tumor location was also significant for DM and OS, with 5-year rates of $69 \%$ vs $33 \%$ and $41 \%$ vs 66\% for trunk vs other locations, respectively. Conclusions: The natural course of a malignant skin melanoma treated radically is disadvantageous, with unsuccessful outcome in nearly half of the cases. Common clinical factors, such as Clark's tumor stage, Breslow's depth of invasion and the presence of metastatic nodes, have high prognostic significance. The size and location of the primary lesion may be considered independent prognostic factors. The most important negative prognostic factor is the presence of metastatic regional lymph nodes. Only one quarter of patients with metastases in lymph nodes survive 5 years from primary surgery.
\end{abstract}

Keywords: Malignant skin melanoma - prognostic factors - radical surgery - distant metastases - tumor diameter

Asian Pac J Cancer Prev, 16 (14), 5709-5714

\section{Introduction}

Malignant skin melanoma is a highly aggressive tumor with an unpredictable clinical course (Thompson et al., 2005; Singluff et al., 2011). A surgical resection with a proper margin is the "gold standard," and it is often accompanied by a surgical excision of the sentinel node (NCCN Guidelines v 3.0 2013; Thompson et al., 2005; Singluff et al., 2011). However, in clinical practice, it is not uncommon for patients to require radicalization after the surgical treatment with margins that are too narrow.

There are many different factors, both tumor- and patient-related, that may influence the progression and prognosis of the disease. The primary risk factors are associated with parameters such as Clark's tumor stage, Breslow's depth of invasion and the presence of metastatic local lymph nodes at the time of initial diagnosis (White et al., 2002; Scaggins et al., 2010; Mervic L, 2012; Teixeira et al., 2013; Deshmane et al., 2014)

Because the clinical course of malignant melanoma is difficult to predict, it seems important to evaluate the clinical factors that may influence the prognosis. Such an assessment on a local population is recommended because the death rates due to malignant skin melanoma among Poles are significantly (up to 20\%) higher than the average for the European Union (Wojciechowska and Didkowska, 2015).

The aim of this paper was to evaluate the results of radical treatment of malignant skin melanoma and to assess the potential factors that may influence the rates of distant metastases (DM), overall survival (OS) and disease-free survival (DFS). 


\section{Materials and Methods}

The retrospective analysis encompasses patients with malignant skin melanoma who were referred to the treatment at the Institute of Oncology in Gliwice between 2008 and 2010. The majority (85\%) of the patients had undergone a prior excision of the primary lesion at other health care centers. According to the recommendations, such surgeries had to be followed with a radicalization (NCCN Guidelines v 3.0, 2015). However, some of the patients were undergoing the entire treatment at the Institute. Patients who had been diagnosed with a local or regional recurrence were excluded from this analysis.

The study group consisted of 60 females and 53 males. All of the patients were in good general health (ECOG $0-1)$. The patients ranged in age from 18 to 82 years old, with an average of 55 years.

The clinical characteristics of the study group are presented in Table 1.

The following factors may have a significant influence on the occurrence of distant metastases, disease-free survival and overall survival and were thus included in the analysis: the patients' gender, location of the primary tumor site (extremities, trunk, head and neck), the surgical margin of the primary surgery (positive or negative), Clark's tumor stage, Breslow's depth of invasion, average size of the primary tumor, ulceration of the tumor, and the presence of metastatic regional lymph nodes ( $\mathrm{pN} 0$ vs $\mathrm{pN}+)$.

\section{Treatment}

In 101 cases, the primary treatment consisted of local excision without lymph node surgery. In the 12 remaining cases, the primary surgery was accompanied by a sentinel node biopsy or lymphadenectomy.

In 20 cases (18\%), the primary surgery was considered to be sufficient. However, in the 93 remaining cases, the patients underwent some type of radicalization. In 19 cases (17\%), a local radicalization was performed, and 4 of these patients also underwent a lymphadenectomy as the third stage. In remaining 74 cases $(83 \%)$, lymphadenectomy was performed accompanied by a local radicalization.

The average period between the primary treatment and the radicalization was 2 months (from 0.1 to 4 months). In $90(80 \%)$ cases, regional lymph node surgery was performed (sentinel node surgery or lymphadenectomy).

A histopathological examination of the tumor excised during the primary surgery allowed for an assessment of Clark's stage in $90 \%$ of the cases, Breslow's depth of invasion in $82 \%$ of the cases and presence of ulceration in $85 \%$ of the cases. An average diameter of the tumor $(a+b / 2)$ could be measured in $89 \%$ of the cases and was $1.7 \mathrm{~cm}$ (median: $1.3 \mathrm{~cm}$; range: from 0.4 to $6 \mathrm{~cm}$ ). In 10 cases $(9 \%)$, the histopathological examination revealed involvement of the margins after the primary surgery. Metastases in regional lymph nodes $(\mathrm{pN}+)$ were discovered in 44 patients $(39 \%)$.

Adjuvant radiotherapy was performed in 48 patients $(30 \%)$. In most of these patients, the clinical target volume (CTV) included regional lymph nodes, which was indicated in the case of lymph node involvement (pN+), In 5 cases, the CTV also included the tumor bed, and in 3 cases, the CTV included only the tumor bed. The radiotherapy was performed with $20 \mathrm{MV}$ and $6 \mathrm{MV}$ $\mathrm{X}$ photons, using 3D conformal or intensity modulated radiotherapy (IMRT) dynamic techniques. The elective volumes were irradiated with 60 Gy total dose (2 Gy per fraction). In case of an involved surgical margin after radicalization, the total dose was increased to approximately $66 \mathrm{~Gy}$.

\section{Statistical analysis}

The statistical analysis of the selected groups was performed using a $\chi^{2}$ test. The survival curves were obtained using the actuarial method from the date of the primary surgery. The study endpoints were overall survival (OS), disease-free survival (DFS) or distant metastases rate $(\mathrm{DM})$. If information on the patient's status was missing, the endpoints were calculated to the date of the last examination of the patient or, as for the OS, to the date of the last information about the patient, which was obtained from the registries or general practitioner. A comparison between groups was performed using the log-rank test. The multivariate analysis, performed using

Table 1. Selected Clinical Characteristics of the Study Group

\begin{tabular}{|c|c|}
\hline Clinical feature & Number of patients (\%) \\
\hline \multicolumn{2}{|l|}{ Gender } \\
\hline Males & $53(47 \%)$ \\
\hline Females & $60(53 \%)$ \\
\hline \multicolumn{2}{|l|}{ Location } \\
\hline Extremities & $51(45 \%)$ \\
\hline Trunk & $44(39 \%)$ \\
\hline Head and neck & $18(16 \%)$ \\
\hline \multicolumn{2}{|l|}{ Clark's tumor stage } \\
\hline I & $1(1 \%)$ \\
\hline II & $13(11 \%)$ \\
\hline III & $47(42 \%)$ \\
\hline IV & $30(26 \%)$ \\
\hline $\mathrm{V}$ & $11(10 \%)$ \\
\hline Unclassified & $11(10 \%)$ \\
\hline \multicolumn{2}{|c|}{ Breslow's depth of invasion (mm) } \\
\hline$<0,75$ & $4(4 \%)$ \\
\hline $0,75-1,5$ & $16(14 \%)$ \\
\hline $1,5-4,0$ & $42(37 \%)$ \\
\hline$>4$ & $30(27 \%)$ \\
\hline Unclassified & $21(18 \%)$ \\
\hline \multicolumn{2}{|c|}{ Average diameter of a tumor } \\
\hline$\leq 1 \mathrm{~cm}$ & $40(35 \%)$ \\
\hline $1-2 \mathrm{~cm}$ & $36(32 \%)$ \\
\hline$>2 \mathrm{~cm}$ & $24(21 \%)$ \\
\hline Unclassified & $13(12 \%)$ \\
\hline \multicolumn{2}{|c|}{ Surgical margin after primary surgery } \\
\hline$(+)$ & $10(9 \%)$ \\
\hline$(-)$ & $103(91 \%)$ \\
\hline \multicolumn{2}{|c|}{ The regional lymph nodes status } \\
\hline $\mathrm{pN}(-)$ & $69(61 \%)$ \\
\hline $\mathrm{pN}(+)$ & $44(39 \%)$ \\
\hline \multicolumn{2}{|l|}{ Tumor ulceration } \\
\hline Absent & $53(47 \%)$ \\
\hline Present & $43(38 \%)$ \\
\hline Unclassified & $17(15 \%)$ \\
\hline
\end{tabular}


Treatment Outcome and Prognostic Factors for Malignant Skin Melanoma Treated with Radical Surgery

the Cox's risk regression analysis, included the variables that achieved a statistical significance of at least $p<0.1$ in the univariate analysis. The hazard ratio (HR) calculated with that model represents the change of the risk of the event (distant metastases, treatment failure or death) with a change of an analyzed parameter.

\section{Results}

The median follow-up was 57 months (range: 5-73 months). During that period, 57 patients (45\%) experienced the following forms of treatment failure: local recurrence (13 cases, $11.5 \%$ ), regional recurrence (17 cases, $15 \%$ ), and distant metastases (40 cases, $35 \%$ ). Of the 30 cases with local or regional recurrence, isolated local recurrence occurred in 6 cases $(5.3 \%)$, and isolated regional recurrence occurred in 5 cases $(4.4 \%)$. Most $(75 \%)$ of the distant metastases manifested themselves during the first two years after treatment completion.

The 3- and 5-year actuarial rates of DM in the study group were $35 \%$ and $47 \%$, respectively. The 3- and 5-year DFS were $51 \%$ and $35 \%$, respectively, and the 3 - and 5 -year OS were $67 \%$ and $56 \%$, respectively. These results are presented in Figures 1 and 2.

In the next stage of the analysis, the effect of the clinical factors on the occurrence of distant metastases, disease-free survival and overall survival were evaluated. The results are presented in Tables 2 and 3.

The results of the treatment were worse in patients who had undergone adjuvant radiotherapy. A 5-year rate

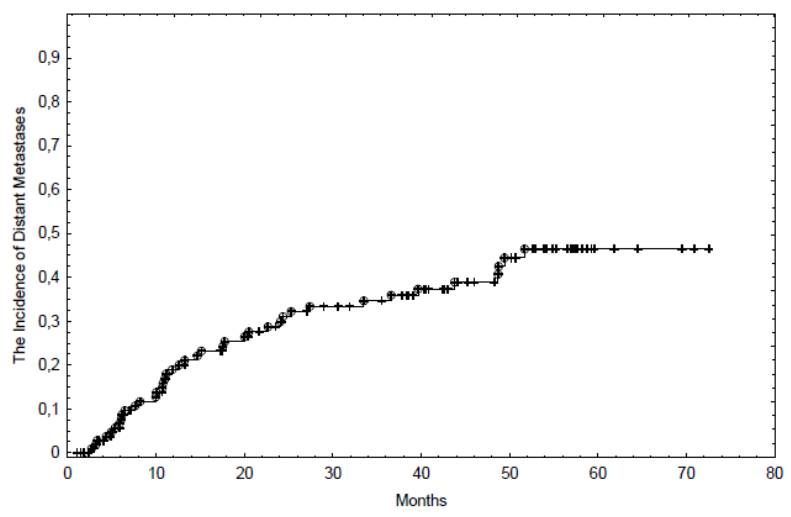

Figure 1. The Actuarial Incidence of Distant Metastases (DM)

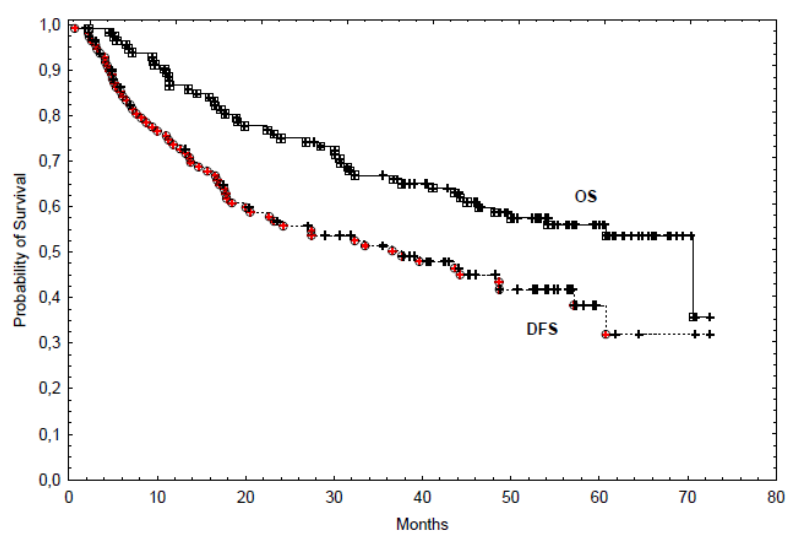

Figure 2. The actuarial Overall Survival and DiseaseFree Survival

of distant metastases was $39 \%$ vs $72 \%(\mathrm{p}=0.006)$, the disease-free survival was $21 \%$ vs $45 \%(\mathrm{p}=0.034)$ and the overall survival was $32 \%$ vs $62 \%(\mathrm{p}=0.008)$. However, the application of adjuvant radiotherapy was related to the presence of additional risk factors, such as a positive surgical margin and the presence of nodal metastases $(\mathrm{pN}+)$. Therefore, the effect of the adjuvant radiotherapy could be reliably estimated only in the multivariate analysis.

Among the group of patients with lymph node metastases, the present analysis did not reveal detailed features related to the prognosis. The number of metastatic nodes, the extracapsular infiltration, and the ratio of metastatic to resected nodes were all not related to DM, DFS or OS in the study group.

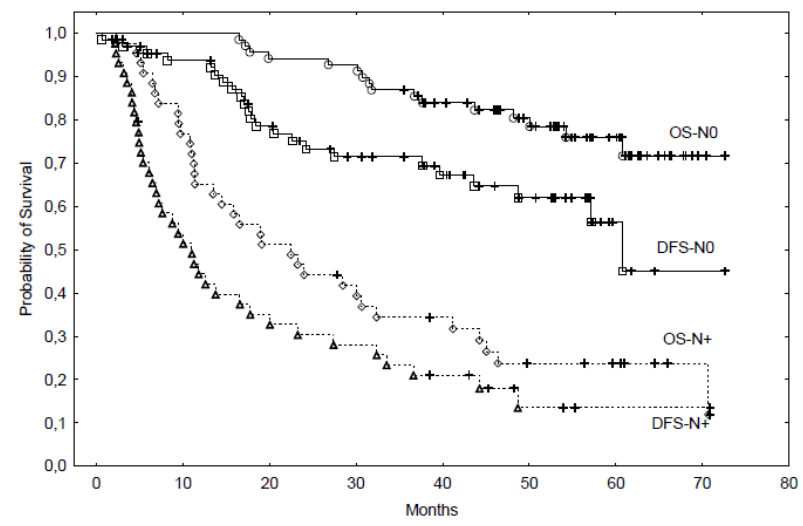

Figure 4. The Overall and Disease-Free Survival with respect to the involvement of regional lymph nodes

Table 2. The Influence of the Selected Factors on the Occurrence of Distant Metastases

\begin{tabular}{|c|c|c|}
\hline Factor & 5-Year Distant Metastases Rate & P Value \\
\hline \multicolumn{2}{|l|}{ Gender } & 0.09 \\
\hline Female & $35 \%$ & \\
\hline Male & $60 \%$ & \\
\hline \multicolumn{2}{|l|}{ Location } & 0.04 \\
\hline Extremities & $29 \%$ & \\
\hline Trunk & $69 \%$ & \\
\hline Head And Neck & $40 \%$ & \\
\hline \multicolumn{2}{|c|}{ Primary Surgery Margin } & 0.22 \\
\hline$(-)$ & $44 \%$ & \\
\hline$(+)$ & $67 \%$ & \\
\hline \multicolumn{2}{|l|}{ Clark's Tumor Stage } & 0.008 \\
\hline I-III & $35 \%$ & \\
\hline IV-V & $60 \%$ & \\
\hline \multicolumn{2}{|c|}{ Breslow's Depth Of Invasion } & 0.004 \\
\hline$<1.5 \mathrm{~mm}$ & $19 \%$ & \\
\hline $1.5-4 \mathrm{~mm}$ & $42 \%$ & \\
\hline$>4 \mathrm{~mm}$ & $72 \%$ & \\
\hline \multicolumn{2}{|c|}{ Average Diameter Of The Tumor1 } & 0.02 \\
\hline$\leq 1 \mathrm{~cm}$ & $28 \%$ & \\
\hline $1-2 \mathrm{~cm}$ & $58 \%$ & \\
\hline$>2 \mathrm{~cm}$ & $64 \%$ & \\
\hline \multicolumn{2}{|c|}{ Regional Lymph Nodes Involvement } & 0.000 \\
\hline $\mathrm{pN}(-)$ & $30 \%$ & \\
\hline $\mathrm{pN}(+)$ & $76 \%$ & \\
\hline \multicolumn{2}{|l|}{ Tumor Ulceration } & 0.01 \\
\hline Absent & $30 \%$ & \\
\hline Present & $58 \%$ & \\
\hline
\end{tabular}


Table 3. The Influence of Selected Factors on the Disease-Free Survival (DFS) and Overall Survival (OS)

\begin{tabular}{|c|c|c|c|c|}
\hline Factor & 5-Year DFS & P Value & 5-Year OS & P Value \\
\hline Gender & & 0.08 & & 0.046 \\
\hline Female & $50 \%$ & & $64 \%$ & \\
\hline Male & $22 \%$ & & $46 \%$ & \\
\hline Location & & 0.16 & & 0.051 \\
\hline Extremities & $48 \%$ & & $67 \%$ & \\
\hline Trunk & $26 \%$ & & $41 \%$ & \\
\hline Head And Neck & $48 \%$ & & $65 \%$ & \\
\hline Primary Surgery Margin & & 0.15 & & 0.11 \\
\hline Negative & $40 \%$ & & $60 \%$ & \\
\hline Positive & $30 \%$ & & $30 \%$ & \\
\hline Clark's Tumor Stage & & 0.015 & & 0.004 \\
\hline I-III & $49 \%$ & & $70 \%$ & \\
\hline IV-V & $30 \%$ & & $40 \%$ & \\
\hline Breslow's Depth Of Invasion & & 0.005 & & 0.002 \\
\hline$<1.5 \mathrm{~mm}$ & $73 \%$ & & $85 \%$ & \\
\hline $1.5-4 \mathrm{~mm}$ & $52 \%$ & & $62 \%$ & \\
\hline$>4 \mathrm{~mm}$ & $20 \%$ & & $35 \%$ & \\
\hline Average Diameter Of The Tumor & & 0.000 & & 0.002 \\
\hline$\leq 1 \mathrm{~cm}$ & $69 \%$ & & $80 \%$ & \\
\hline $1-2 \mathrm{~cm}$ & $28 \%$ & & $53 \%$ & \\
\hline$>2 \mathrm{~cm}$ & $18 \%$ & & $30 \%$ & \\
\hline Regional Lymph Nodes Involvement & & 0.000 & & 0.000 \\
\hline $\mathrm{pN}(-)$ & $56 \%$ & & $76 \%$ & \\
\hline $\mathrm{pN}(+)$ & $13 \%$ & & $24 \%$ & \\
\hline Tumor Ulceration & & 0.048 & & 0.06 \\
\hline Absent & $53 \%$ & & $68 \%$ & \\
\hline Present & $37 \%$ & & $49 \%$ & \\
\hline
\end{tabular}

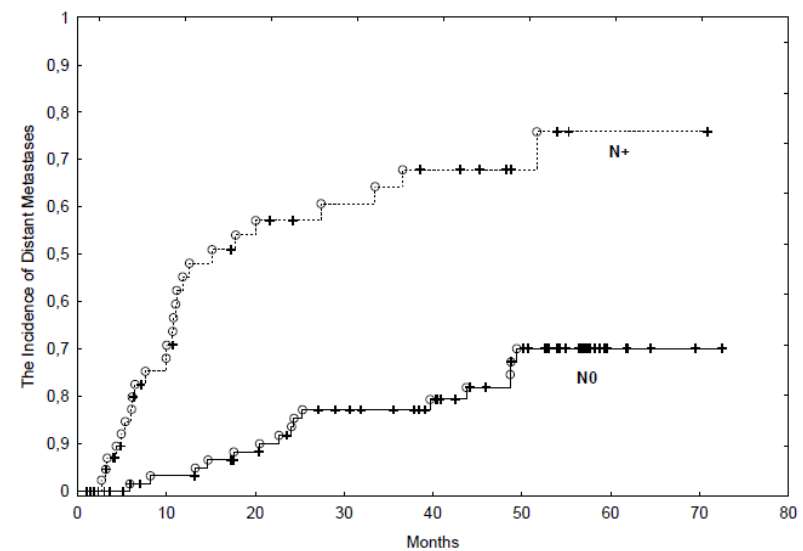

Figure 3. The Incidence of Distant Metastases with Respect to the Involvement of Regional Lymph Nodes

The factors that were included in the multivariate analysis were those that had achieved a statistical significance of at least $\mathrm{p}<0.1$ in a univariate analysis. Additionally, the application of adjuvant radiotherapy was included. The most important factor that had an effect on all endpoints was the presence of nodal metastases (for DM, DFS and OS, respectively: $\mathrm{HR}=3.8 \mathrm{p}=0.002$, $\mathrm{HR}=4.0 \mathrm{p}=0.000, \mathrm{HR}=5.2 \mathrm{p}=0.000$ ). Additionally, the average diameter of the tumor was significant for OS and borderline for DFS with $\mathrm{HR}=1.9, \mathrm{p}=0.03$ and $\mathrm{HR}=1.7$, $\mathrm{p}=0.06$, respectively. The tumor location (trunk $v s$ others) was significant for $\mathrm{DM}$ and $\mathrm{OS}$, with $\mathrm{HR}=3.9, \mathrm{p}=0.002$ and $\mathrm{HR}=2.4, \mathrm{p}=0.01$, respectively. The remaining factors were not independently related to the prognosis in the multivariate analysis. The incidence of the DM, OS and
DFS curves with regard to the most important prognostic factor (metastases in lymph nodes) is illustrated in Figures 3 and 4 .

\section{Discussion}

Of note, in the group of patients with malignant skin melanoma who underwent a radical treatment, the lesion was diagnosed mostly at an intermediate or advanced stage. Additionally, regional lymph nodes metastases were detected in over $1 / 3$ cases.

Our own and others observations also led to a hypothesis that diagnoses of malignant skin melanoma are not efficient and result in detection at an advanced stage [de Vries et al., 2003; Mackie et al., 2009; Gamisizkan et al., 2014). The deficiencies in the diagnostics led to a referral for radical treatment among patients in an advanced stage who had upfront worse prognosis.

Our own research and data from the literature suggest that males have a worse prognosis than do females, including the occurrence, morphologic characteristic of the primary lesion and overall survival (Scoggins et al., 2006; Lasithiotakis et al., 2008; de Vries et al., 2008). Among the probable causes of such results, one could list a more unfavorable natural history, late diagnosis because the first signs of the disease were ignored, or a greater level of sun-induced UV exposition with no UV filters (Scoggins et al., 2006; Lasithiotakis et al., 2008; de Vries et al., 2008; Micheli et al., 2009). The significance of the last probable cause emerges from the results of a study that indicated a correlation between sunbathing habits and the development of the tumor and prognosis for melanoma 
patients (Berwick et al., 2005; Gandini et al., 2013).

Another observation concerns the location of the lesion. The results of the treatment are worse among patients with lesions located on their trunks. It is difficult to provide a single interpretation of this observation. However, because the observed trunk-located lesions were of a greater size than the ones located on extremities, neck or head, one possible explanation would be the greater difficulty in observing the appearance and progression of a lesion by the patients themselves. This may be particularly important with lesions located in non-exposed parts of the body and may be related to a tendency to ignore such lesions. Our own observation has been confirmed by other authors (Garbe et al., 1995). However, it is also possible that worse prognosis for trunk-located tumors may be caused by different biological and clinical features related to tumor location, such as more diversified lymphatic drainage from the trunk. In contrast, other authors have reported worse prognosis for lesions on the extremities than on the trunk (Morton et al., 1991).

The size of the primary tumor has been related to worse prognosis, which has been assessed and confirmed by other authors (Callery et al. 1982; Mervick, 2012; Deshmane et al., 2014). However, this factor is not included in the commonly used classification systems. It is worth emphasizing that the prognostic relevance of the diameter of the tumor was an independent factor in the multivariate analysis. From the biological perspective, the size of the tumor is related to its volume and, thus, to the number of clonogenic cells. This leads to the increased risk of gaining a more aggressive phenotype by tumor cells and, eventually, to a lower TCP (tumor-control probability) ratio (Bentzen, 1996). Therefore, it seems that the diameter of the primary tumor should be considered during the treatment planning phase.

The factor with the most negative prognostic relevance is the presence of metastases in regional lymph nodes. Patients with metastatic nodes are characterized by a 5 -year OS ratio of only approximately $25 \%$, which is three-fold worse than in patients without metastatic nodes. This result suggests that there is a need for more efficient treatment methods for node-positive melanoma patients. Because the predominant cause of treatment failure is distant metastases, the research should focus on a systemic adjuvant treatment. Our own results on that issue are in accordance with the results obtained by other authors, who have indicated that the presence of metastatic nodes is a strong unfavorable prognostic factor (Callery et al., 1982; Garbe et al., 1995; Barnhill et al., 1996; White et al., 2002; Scaggins et al., 2010; Mervic, 2012; Teixeira et al., 2013; Deshmane et al., 2014). We were not able to reveal any detailed features that characterize nodal metastases and are potentially related to prognosis (e.g., node size, extracapsular extension, ratio of number of metastatic to resected nodes). These parameters are often considered important prognostic factors (Callery et al., 1982; Morton et al., 1991; Deshmane et al., 2014), and they have a predictive value by defining the indications for adjuvant radiotherapy (Garbe et al., 1995; Dummer et al. 2012). The present results on that topic might have been caused by a relatively small sample size and the retrospective character of the research. However, even under these circumstances, it was possible to detect many other relationships, which suggests that the study group was homogeneous.

According to some studies, the depth of invasion (thickness) of the tumor may be the most important prognostic factor (Garbe et al., 1995). Other analyses have included the thickness of a tumor, patients' gender, and location of a lesion, and they revealed that these factors significantly influence the prognosis (Callery et al., 1982, Morton et al., 1991; White et al., 2002; Mervic, 2012; Deshmane et al., 2014). The results of this study confirm the relevance of these factors. However, the presence of metastatic lymph nodes seems to be the most negative prognostic factor. It should be noted that the presence of metastatic nodes is not a cause of this worse prognosis itself but is a manifestation of the high metastatic potential of the tumor and its biological aggressiveness. This view is confirmed by dominant cause of treatment failure being: distant metastases (White et al., 2002; Deshmane et al., 2014) and the lack of a clear improvement in overall survival among patients who underwent elective lymphadenectomy or radiotherapy (Lens et al., 2002; Burmeister et al., 2012). This finding suggests that in patients with advanced stages of the disease, even an aggressive locoregional therapy will likely have little influence on the treatment outcome. The weak point of the present analysis is that it does not include modern histopathological factors, such as the mitotic index; however, information on such factors was often lacking due to the retrospective character of the study.

It seems that despite social campaigns related to UV protection, the risks associated with high sunlight and solarium exposure and the significance of consulting every suspicious skin lesion with a physician, the population awareness of the problem is still insufficient (Gajda and Kaminska-Winciorek, 2014; Kaminska-Winciorek et al., 2015). Thus, preventing malignant skin melanoma should be of primary importance, which is augmented by the fact that the natural history of this disease is highly disadvantageous and the radical treatment is unsuccessful in nearly half of the patients.

In conclusion, the natural course of malignant skin melanoma is disadvantageous. Radical treatment is unsuccessful in nearly half of all cases. The common clinical factors, such as Clark's tumor stage, Breslow's depth of invasion and the presence of metastatic nodes, have a high prognostic significance that can be observed even in such small study group. The size and location of the primary lesion may be considered independent prognostic factors. The most important negative prognostic factor is the presence of metastatic regional lymph nodes. Only $1 / 4$ of patients with metastases in lymph nodes survive 5 years from the primary surgery.

\section{References}

Barnhill RL, Fine JA, Roush GA, Berwick M (1996). Predicting five-year outcome for patients with cutaneous melanoma in a population-based study. Cancer, 78, 427-32

Bentzen MS, Thames HD (1996). Tumor volume and local control probability: Clinical data and radiobiological 
Wojciech Majewski et al interpretations. Int J Radiation Oncology Biol Phys, 36, 247-51

Berwick M, Armstrong BK, Ben-Porat L, et al (2005). Sun exposure and mortality from melanoma. $J$ Natl Cancer Inst, 97, 195-9

Burmeister BH, Henderson MA, Ainslie J et al (2012). Adjuvant radiotherapy versus observation alone for patients at risk of lymph node field relapse after therapeutic lymphadenectomy for melanoma: a randomised trial. Lancet Oncol, 13, 589-97

Callery Ch, Cochran AJ, Roe DJ, et al (1982). Factors prognostic for survival in patients with malignant melanoma spread to the regional lymph nodes. Ann Surg, 196, 69-75

Deshmane V, Kalloli M, Chikaraddi S, et al (2014). Predictive factors for loco regional recurrence and distant metastasis following primary surgical treatment of cutaneous melanoma. Indian J Dermatol, 59, 241-6

Dummer R, Hauschild A, Guggenheim M, et al (2012). Cutaneous melanoma: ESMO Clinical Practice Guidelines for diagnosis, treatment and follow-up. Ann Oncol, 23, 86-91

Gajda M, Kaminska-Winciorek G (2014). Do not let to be late: Overview of reasons for melanoma delayed diagnosis. Asian Pac J Cancer Prev, 15, 3873-7

Gamsizkan M, Yilmaz I, Buyukbabani N, et al (2014). A retrospective multicenter evaluation of cutaneous melanomas in Turkey. Asian Pac J Cancer Prev, 15, 10451-6

Gandini S, De Vries E, Tosti G, et al (2013). Sunny holidays before and after melanoma diagnosis are respectively associated with lower breslow thickness and lower relapse rates in italy. Plos One, $\mathbf{8}$.

Garbe C, Buttner P, Bertz J et al (1995). Primary cutaneous melanoma. Identification of prognostic groups and estimation of individual prognosis for 5093 patients. Cancer, 75, 2484-91

Garbe C, Buttner P, Bertz J, et al (1995). Primary cutaneous melanoma. prognostic classification of anatomic location. Cancer, 75, 2492-8.

Kaminska-Winciorek G, Gajda M, Wydmanski J, Tukiendorf A (2015). What do web users know about skin self-examination and melanoma symptoms. Asian Pac J Cancer Prev, 16, 3051-6

Lasithiotakis K, Leiter U, Meier F, et al (2008). Age and gender are significant independent predictors of survival in primary cutaneous melanoma. Cancer, 112, 1795-804.

Lens MB, Dawes M, Goodacre T, Newton-Bishop JA (2002). Elective lymph node dissection in patients with melanoma: systematic review and meta-analysis of randomized controlled trials. Arch Surg, 137, 458-61

Mackie RM, Hauschild A, Eggermont AM (2009). Epidemiology of invasive cutaneous melanoma. Ann Oncol, 20, 1-7

Melanoma. NCCN clinical practice guidelines in oncology. Version, 3, 2-24.

Mervic L (2012). Prognostic factors in patients with localized primary cutaneous melanoma. Acta Dermatovenerol APA, 21, 27-31

Micheli A, Ciampichini R, Oberaigner W, et al (2009). The advantage of women in cancer survival: an analysis of EUROCARE-4 data. Eur J Cancer, 45, 1017-27

Morton DL, Wanek L, Nizze JA, et al. (1991). Improved long-term survival after lypmhadenectomy of melanoma metastatic to regional nodes. Ann Surg, 214, 491-9

Scoggins CR, Ross MI, Reintgen DS, et al (2006). Genderrelated differences in outcome for melanoma patients. Ann Surg, 243, 693-8

Scoggins CR, Bowen AL, Martin RC II, et al (2010). Prognostic information from sentinel lymph node biopsy in patients with thick melanoma. Arch Surg, 145, 622-7

Slingluff CL, Flaherty K, Rosenberg SA, Read PW (2011).
Cutaneous melanoma. In De Vita VT, Lawrence TS, Rosenberg SA. Cancer Principles PractOncol. Lippincott, Wiliams and Wilkins

Teixeira V, Vieira R, Coutinho I, et al (2013). Prediction of sentinel node status and clinical outcome in a melanoma centre. J Skin Cancer. 2013, 904701

Thompson JF, Scolyer RA, Kefford RF (2005). Cutaneous melanoma. Lancet, 365, 687-701

White RR, Stanley WE, Johnson JL, et al (2002). Long-Term Survival in 2,505 Patients With Melanoma With Regional Lymph Node Metastasis. Ann Surg, 235, 879-87

de Vries E, Bray FI, Coebergh JW, Parkin DM (2003). Changing epidemiology of malignant cutaneous melanoma in Europe 1953-1997: Rising trends in incidence and mortality but recent stabilizations in western Europe and decreases in Scandinavia. Int J Cancer, 107, 119-26

de Vries E, Nijsten TE, Visser O, et al (2008). Superior survival of females among 10,538 Dutch melanoma patients is independent of Breslow thickness, histologic type and tumor site. Ann Oncol, 19, 583-9

Wojciechowska U, Didkowska J (2015), Zachorowania I zgony na nowotwory zlosliwe w Polsce. Krajowy Rejestr Nowotworow www.onkologia.org.pl, 